McGill/93-8

TPI-MINN-93/19-T

\title{
Lepton pairs from thermal mesons
}

\author{
Charles Gale日 \\ Physics Department, McGill University \\ 3600 University St., Montréal, QC, H3A 2T8, Canada \\ Peter Lichardi \\ Theoretical Physics Institute, University of Minnesota \\ Minneapolis, MN 55455, USA \\ and \\ Department of Theoretical Physics, Faculty of Mathematics and Physics \\ Comenius University, CS-842 15 Bratislava, Slovakid
}

\begin{abstract}
We study the net dielectron production rates from an ensemble of thermal mesons, using an effective Lagrangian to model their interaction. The coupling between the electromagnetic and the hadronic sectors is done through the vector meson dominance approach. For the first time, a complete set of light mesons is considered. We include contributions from decays of the type $\mathrm{V}(\mathrm{PS}) \rightarrow \mathrm{PS}(\mathrm{V})+e^{+} e^{-}$, where $\mathrm{V}$ is a vector meson and $\mathrm{PS}$ is a pseudoscalar, as well as those from binary reactions $\mathrm{PS}+\mathrm{PS}, \mathrm{V}+\mathrm{V}$, and $\mathrm{V}+\mathrm{PS} \rightarrow e^{+} e^{-}$. Direct decays of the type $\mathrm{V} \rightarrow e^{+} e^{-}$are included and shown to be important. We find that the dielectron invariant mass spectrum naturally divides in distinct regions: in the low mass domain the decays from vector and pseudoscalar mesons form the dominant contribution. The pionpion annihilation and direct decays then pick up and form the leading signal in an invariant mass region that includes the $\rho-\omega$ complex and extends up to the $\phi$. Above invariant mass $M \approx 1 \mathrm{GeV}$ other two-body reactions take over as the prominent mechanisms for lepton pair generation. These facts will have quantitative bearing on the eventual identification of the quark-gluon plasma.
\end{abstract}

PACS numbers: 25.75.+r, 12.38.Mh, 13.75.Lb

Typeset using REVTEX 


\section{INTRODUCTION}

One of the ultimate goals of high energy heavy ion physics is the formation and observation of a quark-gluon plasma (QGP) as predicted by QCD. A vigorous experimental program is under way and it is fair to say that this area is one of the most active fields of contemporary subatomic physics. The creation of such a novel state of matter represents a considerable challenge, both in its experimental realization and also in the theoretical interpretation of the experimental results. The lifetimes involved are of the order of $\approx 10 \mathrm{fm} / \mathrm{c}$ and the detailed dynamics of the collision process may furthermore play an important role, complicating the extraction of a clear signal. Nevertheless, much progress has been made both in theory and in experiment, and we may say that even in the absence of a genuine QGP the study of hot and dense hadronic systems is still a fascinating subject from which a great deal can be learnt.

For a while now, electromagnetic signals have been known as ideal probes of strongly interacting matter at high temperatures and densities [1]. This owes to the fact that once they are produced, they will travel relatively unscathed from their point of origin to the detector. Since production rates are rapidly increasing functions of temperature and density, these electromagnetic signals provide valuable information on the hot and dense phases of the reaction. It is hoped that, because of these facts, those signals should constitute precious aids in the process of analyzing the behaviour of hot quark-gluon matter [2]. As with any possible experimental signature of the QGP, a great deal of care must go into the calculation of a corresponding "purely hadronic" signal, that is a contribution to the same experimental observables from sources other than the deconfined, chiral-symmetric phase. As far as the quark-gluon plasma is concerned, one may refer to these sources as the "background".

In this paper, we are concerned with the thermal rate of dielectron emission only but our treatment is completely general. The source is a hot environment of several meson species: for the first time, we use a rather complete set of mesons, rather than restricting ourselves to the usual pion gas approximation. The equilibrium assumptions inherent to the approaches similar to the one been used here have to be carried to their logical conclusion: in such scenarios, once the temperature has been set one can clearly calculate the population of species present. These mesons can then interact among themselves, or even decay, to produce lepton pairs in the final state. It is important to realize that we deliberately make no attempt here to connect with experiment because our calculation is rather meant to answer a well defined theoretical question: what is the electromagnetic emissivity (in the dilepton channel) of a hot hadron gas? To answer this question, we shall proceed along the lines of a similar calculation for photon rates [3].

We estimate the rates of producing lepton pairs using relativistic kinetic theory. The mesonic interactions are modelled with an effective Lagrangian and the coupling of radiation to hadronic matter is done in the vector meson dominance (VMD) approach. The values of the coupling constants involved are adjusted so that the experimentally measured radiative decay widths are reproduced. We describe the details of our model in section $\mathbb{1 1}$. We give results in section $\mathbb{I I}$ and finally we end with a discussion in section $\mathbb{}$. 


\section{THE MODEL}

Our starting point is an ensemble of mesons in thermal equilibrium. We consider the lightest and thus most abundant strange and non-strange mesons together with their main interaction channels. This means we shall include: $\pi, \eta, \rho, \omega, \eta^{\prime}, \phi, K$ and $K^{*}$. The charge states are not labelled but all of them are present. This collection can be further divided in two categories: pseudoscalar (PS) and vector (V) particles. From this hot meson gas, how do we calculate what is the lepton pair radiation? It has been shown [4] that the thermal production rate for electron-positron pairs is related to the imaginary part of the retarded photon self energy by

$$
\begin{aligned}
E_{+} E_{-} \frac{d R}{d^{3} p_{+} d^{3} p_{-}}= & \frac{2 e^{2}}{(2 \pi)^{6}} \frac{1}{M^{4}}\left(p_{+}^{\mu} p_{-}^{\nu}+p_{+}^{\nu} p_{-}^{\mu}-p_{+} \cdot p_{-} g^{\mu \nu}\right) \operatorname{Im} \Pi_{(\gamma) \mu \nu}^{\mathrm{R}}(k) \\
& \times \frac{1}{e^{E / T}-1}
\end{aligned}
$$

Here $p_{+}$and $p_{-}$are the positron and electron momenta, $k^{\mu}=(E, \vec{k})$ is the virtual photon momentum, $T$ is the temperature, and we have set the electron mass to zero (nonzero lepton mass is easy to include). $R$ is the number of times per unit four-volume an $e^{+} e^{-}$pair of invariant mass $M$ is produced with the specified momentum configuration. Note that the above equation is perturbative in the electromagnetic interaction only; it is a completely non-perturbative expression in the strong interaction.

Furthermore, we shall make use of the VMD model, which states that the hadronic electromagnetic current operator is given by the current-field identity

$$
J_{\mu}=-\frac{e}{g_{\rho}} m_{\rho}^{2} \rho_{\mu}-\frac{e}{g_{\phi}} m_{\phi}^{2} \phi_{\mu}-\frac{e}{g_{\omega}} m_{\omega}^{2} \omega_{\mu}
$$

The above expression tells us how the electromagnetic radiation couples to hadronic (in our case mesonic) matter: by first coupling to one of the vector mesons with some coupling constant. In the above, we have kept the $\rho, \omega$ and $\phi$ fields, but in some cases we shall tacitly include also higher vector mesons by using phenomenological form factors inspired by data. We further need a model for how the mesons interact among themselves. For this, we shall use a simple phenomenological approach, inspired by the chiral properties of low energy QCD. Such classes of phenomenological Lagrangians have been quite successful in the past in the description of low energy hadronic physics [5]. We are explicitly interested in the interaction between the different possible combinations of vector $(V)$ and pseudoscalar $(\varphi)$ fields. For reasons that will become clear shortly we restrict our discussion to the following interaction Lagrangians [5]:

$$
\mathcal{L}_{V V \varphi}^{\text {int }}=g_{V V \varphi} \epsilon_{\mu \nu \alpha \beta} \partial^{\mu} V^{\nu} \partial^{\alpha} V^{\beta} \varphi
$$

and

$$
\mathcal{L}_{V \varphi \varphi}^{\text {int }}=g_{V \varphi \varphi} V_{\mu} \varphi \stackrel{\leftrightarrow}{\partial} \varphi
$$

In the above, the coupling constants are fitted for each field combination, in a procedure we now describe. We have a model for how mesons interact among themselves and how they 
interact with the electromagnetic field. With this approach, let us study a simple radiative process like the decay of a vector meson into a pseudoscalar meson and a photon like e.g. $\omega \rightarrow \pi^{0} \gamma$. In this model, the process goes via the $\omega \rho \pi$ vertex, owing to $G$ parity conservation at the strong vertex, and the $\rho^{0}$ couples to the photon in virtue of the current-field identity. This corresponds to the Feynman diagram of Fig. 1. The ratio of coupling constants from Eqs. (2.3) and (2.2), in this case $g_{\omega \rho \pi} / g_{\rho}$, is adjusted so that the correct experimental radiative decay width [6] $\Gamma\left(\omega \rightarrow \pi^{0} \gamma\right)$ is obtained. Our Lagrangians are then "calibrated" through all the following processes: $\rho \rightarrow \pi \gamma, K^{* \pm} \rightarrow K^{ \pm} \gamma, K^{* 0}\left(\bar{K}^{*}\right) \rightarrow K^{0}\left(\bar{K}^{0}\right) \gamma$, $\omega \rightarrow \pi^{0} \gamma, \rho^{0} \rightarrow \eta \gamma, \eta^{\prime} \rightarrow \rho^{0} \gamma, \eta^{\prime} \rightarrow \omega \gamma, \phi \rightarrow \eta \gamma, \phi \rightarrow \eta^{\prime} \gamma, \phi \rightarrow \pi^{0} \gamma$. One realizes $[$ c.f. Eqs. (2.2, 2.3, 2.4)] that via this procedure, we can only fix the ratio of strong to "electromagnetic" (vector meson-photon) couplings. However it is this very combination we shall need for our specific application.

We now integrate our model for interacting mesons with a dilepton radiation calculation. If we keep a calculation of the photon self-energy at the one-loop level an evaluation of its imaginary part, as instructed in Eq. (2.1), will yield processes of the type V (PS) $\rightarrow$ PS (V) $\gamma^{*}, \mathrm{PS}+\mathrm{PS} \rightarrow \gamma^{*}, \mathrm{~V}+\mathrm{PS} \rightarrow \gamma^{*}$ and $\mathrm{V}+\mathrm{V} \rightarrow \gamma^{*}$. Since such tree-level amplitudes can be readily computed and that our general field-theoretic treatment for dilepton emission has been shown to agree with relativistic kinetic calculations (up to temperature-dependent effects in the form factors, which have been shown to be small [4]) we use the latter approach. Finally note that the two-body channels listed above will kinematically dominate the contributions of the type $\mathrm{V}+\mathrm{PS} \rightarrow \mathrm{PS}+\gamma^{*}$, which we shall neglect. The inclusion of such processes would correspond to evaluation of the photon self-energy beyond the oneloop level. The first attempt to investigate the role of processes with more than two mesons involved has recently been made in [7]. We will return to this point later.

The basic relativistic kinetic expression for the dilepton production rate from a process $a+b \rightarrow e^{+} e^{-}$is well known and can be written down as

$$
\begin{aligned}
R_{a b \rightarrow e^{+} e^{-}}= & \mathcal{N} \int \frac{d^{3} p_{a}}{2 E_{a}(2 \pi)^{3}} \frac{d^{3} p_{b}}{2 E_{b}(2 \pi)^{3}} \frac{d^{3} p_{+}}{2 E_{+}(2 \pi)^{3}} \frac{d^{3} p_{-}}{2 E_{-}(2 \pi)^{3}} f_{a} f_{b} \\
& \times|\mathcal{M}|^{2}(2 \pi)^{4} \delta^{4}\left(p_{a}+p_{b}-p_{+}-p_{-}\right) .
\end{aligned}
$$

Similarly, we may write a rate equation for the decay process $a \rightarrow b+e^{+} e^{-}$:

$$
\begin{aligned}
\frac{d R_{a \rightarrow b+e^{+} e^{-}}}{d M^{2}}= & \mathcal{N} \int \frac{d^{3} p_{a}}{2 E_{a}(2 \pi)^{3}} \frac{d^{3} p_{b}}{2 E_{b}(2 \pi)^{3}} \frac{d^{3} p_{+}}{2 E_{+}(2 \pi)^{3}} \frac{d^{3} p_{-}}{2 E_{-}(2 \pi)^{3}} f_{a}\left(1+f_{b}\right) \\
& \times|\mathcal{M}|^{2}(2 \pi)^{4} \delta^{4}\left(p_{a}-p_{b}-p_{+}-p_{-}\right) \delta\left(M^{2}-\left(p_{+}+p_{-}\right)^{2}\right) .
\end{aligned}
$$

In the above equations, $\mathcal{N}$ is an overall degeneracy factor dependent upon the specific channel and the $f$ 's are Bose-Einstein mean occupation numbers.

These equations are not suitable for numerical evaluation because of the delta functions. However, they can be cast, using standard methods of simplifying phase integrals and the spherical symmetry in momentum space, into an appropriate form. The dilepton production rate for the process $a+b \rightarrow e^{+} e^{-}$becomes

$$
R_{a b \rightarrow e^{+} e^{-}}=\frac{\mathcal{N}}{(2 \pi)^{4}} \int_{m_{a}}^{\infty} d E_{a} p_{a} f_{a}\left(E_{a}\right) \int_{m_{b}}^{\infty} d E_{b} p_{b} f_{b}\left(E_{b}\right)
$$




$$
\times \int_{-1}^{1} d x \sqrt{\left(s-s_{+}\right)\left(s-s_{-}\right)} \sigma_{a b \rightarrow e^{+} e^{-}}(s),
$$

where $s \equiv M^{2}=m_{a}^{2}+m_{b}^{2}+2\left(E_{a} E_{b}-p_{a} p_{b} x\right), s_{+}=\left(m_{a}+m_{b}\right)^{2}$ and $s_{-}=\left(m_{a}-m_{b}\right)^{2}$. The cross section $\sigma_{a b \rightarrow e^{+} e^{-}}(s)$, is obtained by an evaluation of an appropriate Feynman diagram, Fig. 2. The multiple integral in Eq. (2.7) is evaluated by Monte Carlo methods. While it is certainly possible to do some of the integrations analytically, we chose the avenue of keeping a relatively transparent integrand and we let the Monte Carlo approach handle the numerical complexity. Moreover, this approach allows us to evaluate any desirable differential dilepton production rate easily (see, e.g., [8] or [9]).

Similarly, the rate equation for the decay process $a \rightarrow b+e^{+} e^{-}$is now:

$$
\begin{aligned}
\frac{d R_{a \rightarrow b+e^{+} e^{-}}}{d M^{2}}= & \frac{\mathcal{N} m_{a}}{(2 \pi)^{2}} \frac{d \Gamma_{a} \rightarrow b+e^{+} e^{-}}{d M^{2}} \int_{m_{a}}^{\infty} d E_{a} p_{a} f_{a}\left(E_{a}\right) \\
& \times \int_{-1}^{1} d x\left[1+f_{b}\left(E_{b}\right)\right]
\end{aligned}
$$

where $E_{b}=\left(E_{a} E_{b}^{*}+p_{a} p_{b}^{*} x\right) / m_{a}, E_{b}^{*}=\left(m_{a}^{2}+m_{b}^{2}-M^{2}\right) /\left(2 m_{a}\right)$ and $d \Gamma_{a} \rightarrow b+e^{+} e^{-} / d M^{2}$ is the differential decay width into the appropriate channel. In Eqs. (2.6) and (2.8) one notices the Bose-Einstein final state enhancement, an in-medium effect.

We also include the direct decay channels of the form $V \rightarrow e^{+} e^{-}$. As we will show, their contributions are non-negligible. This is especially true in the case of $\rho \rightarrow e^{+} e^{-}$. One can show that for such decays

$$
\frac{d R_{V \rightarrow e^{+} e^{-}}}{d M^{2}}=\frac{3}{2 \pi^{2}} \frac{\Gamma_{V \rightarrow e^{+} e^{-}}}{\tilde{N}} \frac{m_{V}^{3}}{M^{2}} B\left(M^{2}\right) \int_{M}^{\infty} d E f(E) \sqrt{E^{2}-M^{2}},
$$

where

$$
B\left(M^{2}\right)=\beta \frac{\Gamma_{\text {tot }}}{\left(M^{2}-m_{V}^{2}\right)^{2}+\left(m_{V} \Gamma_{\text {tot }}\right)^{2}} .
$$

The constant $\beta$ fixes the normalization of the Breit-Wigner probability density function. Its value is not important here as it enters also the factor

$$
\tilde{N}=\int d M^{2}\left(\frac{m_{V}}{M}\right)^{3} B\left(M^{2}\right)
$$

which ensures the correct overall normalization based on the experimental value of the partial decay width into the dielectron channel, $\Gamma_{V} \rightarrow e^{+} e^{-}$. The integral runs over the allowed mass range.

In the above equations, $m_{V}$ is the vector meson mass and $\Gamma_{\text {tot }}$ is its total decay width. For the narrow resonances $(\omega, \phi)$ the latter is taken constant but the $\rho^{0}$ width is given its proper mass dependence.

\section{RESULTS}

The decay channels considered have already been listed: they are the same radiative decay reactions $\mathrm{V}(\mathrm{PS}) \rightarrow \mathrm{PS}(\mathrm{V})+\gamma$, as used to fix the couplings constants of our Lagrangians, with the obvious substitution: $\gamma \rightarrow \gamma^{*}$. The $\mathrm{V}+\mathrm{PS} \rightarrow e^{+} e^{-}$amplitudes can all 
be obtained from the decay reaction amplitudes by crossing symmetry. We list the entrance channels anyway for completeness. They are: $\omega \pi^{0}, \rho \pi, \phi \pi^{0}, \omega \eta, \phi \eta, \rho^{0} \eta, \omega \eta^{\prime}, \phi \eta^{\prime}$, $\rho \eta^{\prime}, \bar{K}^{*} K$ and $K^{*} \bar{K}$. For each of the PS + PS and $\mathrm{V}+\mathrm{V}$ reaction, we follow the following approach: their "bare" amplitude is calculated, squared, and finally multiplied by a form factor obtainable from experimental data on $e^{+} e^{-}$annihilation.

The topic of form factors deserves here a short discussion. Of course no information on time-like form factors is available through the analysis of meson radiative decays into real photons. With respect to this issue, we have followed a simple prescription. The timelike electromagnetic form factor of charged pion is experimentally very well known [10] and some experimental information exists also about those of both charged and neutral kaons [11]. In our calculations of $\pi^{+} \pi^{-}, K^{+} K^{-}$, and $K^{0} \bar{K}^{0}$ annihilation rates we have used a recent parametrization [12] of these quantities. The vector mesons annihilation channels have been given the same form factors as their corresponding (by strangeness and isospin) pseudoscalar counterparts. In the case of decays and V + PS reactions, whenever the $G$ parity and isospin conservation laws allowed a coupling only to the $\rho^{0}$ and its recurrences, the charged pion electromagnetic form factor [12] was used. In the other cases, we have stuck with a form factor equivalent to a simple pole corresponding to the lightest permitted vector meson. Our way of normalizing coupling constants by means of the radiative decay widths leads us to a belief that this conservative choice of form factors does not introduce too much uncertainty. We made only one exception from the simple rules sketched above. In the case of the reaction $\rho+\pi \rightarrow e^{+} e^{-}$the rules would lead to a simple $\omega$-pole. It would be a rather bad approximation because the threshold of this reaction lies below the position of the $\phi$-resonance, which thus becomes extremely important. We take therefore a two-pole formula with the relative weight between the $\omega$ and $\phi$ contributions same as in the kaon isoscalar form factor $F_{S}=\left(F_{K^{+}}+F_{K^{-}}\right) / 2$ [12].

We have performed our thermal hadronic calculations at three temperatures: 100, 150 and $200 \mathrm{MeV}$. We feel that those reflect a range of energies that is somewhat reasonable, by current theoretical standards.

The results for $\mathrm{V}(\mathrm{PS}) \rightarrow$ PS $(\mathrm{V}) e^{+} e^{-}$at a temperature of $150 \mathrm{MeV}$ are shown on Fig. 3. Not all the decays are shown, but only the dominant ones. Coupling constants arguments aside, the largest contributions will come from the radiative channels where a heavy meson decays into a light one and a lepton pair. This is precisely what is observed on Fig. 3. The largest contribution up to invariant mass $\approx 0.65 \mathrm{GeV}$ is from $\omega \rightarrow \pi^{0} e^{+} e^{-}$. Over this range, $\rho \rightarrow \pi e^{+} e^{-}$represents the next-to-leading contribution and the other decays are at least an order of magnitude lower. Above $0.65 \mathrm{GeV}$ invariant mass, the only decay with phase space left is $\phi \rightarrow \pi^{0} e^{+} e^{-}$. Note that the widths for the radiative decays of the $\omega$ and $\rho^{0}$ are comparable and are two orders of magnitude larger than that for $\phi \rightarrow \pi^{0} \gamma[6]$. Dalitz decay (e.g. $\left.\eta \rightarrow \gamma e^{+} e^{-}\right)$is of higher order in $\alpha$ and can thus be neglected. However, this argument alone is not totally convincing as one could imagine that the $\eta$ could be massively produced at such temperatures. We have therefore performed a calculation of the contribution from eta Dalitz decay to thermal electron pair yield, using the VMD prescription for $d \Gamma / d M^{2}$ [13] with updated coupling constants. We have found it in fact to be orders of magnitude smaller than the channels discussed above.

For the pseudoscalar-pseudoscalar reactions, on Fig. 4 we display a plot of all the contributions, again at $T=150 \mathrm{MeV}$. The different contributions add up to a signal in 
which the only apparent structures are associated with the $\rho(770)$ and the $\phi$, with a slight shoulder at the $\rho(2150)$. The peak in the pion form factor at the $\rho(1700)$ is washed out by the kaon contributions.

We show the $\mathrm{V}+\mathrm{V}$ contributions on Fig. 5. Above threshold, the sum of these processes outshine the PS + PS ones by roughly an order of magnitude. The structure at $M=2.15$ $\mathrm{GeV}$ owes to the corresponding excitation of the $\rho$.

The $\mathrm{V}+\mathrm{PS}$ reactions are quite numerous, we show the brighter dilepton sources on Fig. 6 , again for $T=150 \mathrm{MeV}$. The dominant channels are $\omega+\pi^{0}, \rho+\pi$ and $\rho^{0}+\eta$. The kaon channels are not shown but are roughly the size of the $\pi+\rho$ contribution. The strongest signal is from $\omega+\pi^{0}$, over the entire invariant mass range considered here. Recall from our discussion of the decays that the radiative decay widths of the $\rho$ and the $\omega$ are quite large.

Finally, the total rate corresponding to the sum of all processes discussed so far is shown on Fig. 7, along with a curve representing the $\pi^{+} \pi^{-}$contribution only. We also show the net direct decay contribution, summing $\rho \rightarrow e^{+} e^{-}, \omega \rightarrow e^{+} e^{-}$and $\phi \rightarrow e^{+} e^{-}$. The radiation from these channels turns out to be quite important. The signal from the decay reaction $\rho \rightarrow e^{+} e^{-}$closely resembles the pion annihilation spectrum, which in retrospect is quite reasonable.

In all cases (decays, PS $+\mathrm{PS}, \mathrm{V}+\mathrm{V}, \mathrm{V}+\mathrm{PS}$ ) our findings at $T=100$ and $200 \mathrm{MeV}$ are qualitatively similar, with a global shift in the rate. For these temperatures we therefore present only the total rates (see Fig. 8).

\section{DISCUSSION}

Up to now, thermal calculations of the variety discussed in this paper have rarely gone beyond a pure pion gas approximation, usually concentrating on the annihilation channel [14]. The contribution from thermal meson decays has been considered previously [15]. To our knowledge it is the first time that extensive mesonic reactions have been included, together with direct decays.

Comparing the individual contributions from different processes (Figs. 3-6) to the total dilepton rate (Fig. 7) one sees that the dilepton invariant mass spectrum naturally divides in several parts. At low masses, the decay channels clearly dominate the entire spectrum. The crossover to the pion-pion annihilation and direct decay signal occurs just above 0.5 $\mathrm{GeV}$ (at the lower temperature, $T=100 \mathrm{MeV}$, this crossing point is shifted closer to the two-pion threshold). Already at $M \approx 1 \mathrm{GeV}$, the total rate dominates over the pion gas approximation result by an approximate factor of 3 . At $M=1.5 \mathrm{GeV}$, those rates differ by a little more than an order of magnitude. The difference increases with larger invariant masses. One also sees that the net rate at the vector meson positions is also larger than in the straight $\pi^{+}-\pi^{-}$scenario, owing principally to direct decays and also form factor effects. Probably the most striking conclusion of our work is that the "usual" pion results for lepton pair production calculation holds rather poorly over all regions of invariant masses considered in this work. This statement is true for all temperatures studied here.

Thus, the rate for $M \gtrsim 1 \mathrm{GeV}$ is approximately one order of magnitude larger in our calculation than in "conventional" meson background calculations. This enhancement is also present in the momentum structure of the lepton signal: Fig. 9 is a plot of $E d^{3} R / d^{3} p$ for 
lepton pair invariant masses between 1.1 and $3 \mathrm{GeV}$. These findings should have important implications in connection with the plasma signal identification. The conventional window for thermal lepton pairs of plasma origin is $m_{\phi}<M<m_{J / \psi}$ [2], precisely the range discussed here. An observation of a signal from an exotic source can only be claimed if all other sources are under control. Here these would be identified with the Drell-Yan mechanism, open charm decay [16] and the thermal background we have considered in this work. However, before any more quantitative statements can be made, it is imperative to complement our calculations with a dynamical model of some sort, in order to make contact with genuine observables. Work in this direction is in progress.

It is of interest to compare the rates obtained with other similar calculations. Some recent interest has been devoted to the emission of lepton pairs from pionic bremsstrahlung processes [17]. It was concluded that the radiation from the external pion lines in pion-pion collisions would be a dominant contribution to the low mass lepton spectrum. Comparing with pion bremsstrahlung calculations at $T=150 \mathrm{MeV}$, we realize that the low mass signal is the same magnitude as the net meson decay contribution. Correcting the pion-pion bremsstrahlung rate for the Landau-Pomeranchuk effect 18 will cut this pion signal by some factor. This factor is only $\approx 2$ for low invariant masses and $T=150 \mathrm{MeV}$ [19]. This correction also goes down as invariant mass grows. This will then leave the pion bremsstrahlung to compete with the decay channels contribution, up to the two-pion annihilation threshold.

In this inquiry, we have pursued the same goals as a similar photon production calculation [3]. Our main aim has been to identify the most important dilepton production processes which operate in a hadron gas. We considered only the decays and reactions with the minimal possible number of hadrons: one in the decay final states, none in the final states of two-initial-hadron reactions. These processes are believed, on the basis of the phasespace and order-of-interaction arguments, to be dominant here. The reactions of this kind $(2 \rightarrow 0$ hadrons $)$ do not operate in real photon production due to restrictions from energymomentum conservation. However, the dominant reactions for photon production $a+b \rightarrow$ $c+\gamma$ can produce virtual photons as well. It is clear that they would populate preferably the low-mass region. Even there they would be probably negligible, as it was shown for the case of $\pi+\pi \rightarrow \pi+$ dilepton in [7]. But one cannot exclude surprises. The latter process amplifies, together with the three pion annihilation channel, the omega peak in dilepton spectrum. This may in turn serve as a signature of a hadron gas creation [7]. It has also been pointed out that the $A_{1}$ meson could have a significant influence on the real photon yield [20], through the process $\pi \rho \rightarrow A_{1} \rightarrow \pi \gamma$. This conjecture has been carefully analyzed in a recent paper [21]. The reflection in the thermal dilepton sector is certainly worth studying as well. Three body initial state processes $a+b+c \rightarrow e^{+} e^{-}$may also contribute significantly in the high invariant mass region [7]. We intend to study all these points in detail in future work.

\section{ACKNOWLEDGMENTS}

We would like to acknowledge the warm hospitality of the Theoretical Physics Institute of the University of Minnesota, where this work was started. This work was supported in part by the Natural Sciences and Engineering Research Council of Canada, by the FCAR 
fund of the Québec Government and by a NATO Collaborative Research grant. The stay of P.L. at the University of Minnesota was supported by the U.S. Department of Energy under Contract No. DOE/DE-FG02-87ER-40328; travel expenses were borne by the grant MŠMŠ SR 01/35. 


\section{REFERENCES}

* e-mail: gale@physics.mcgill.ca

$\dagger \quad$ e-mail: lichard@physics.spa.umn.edu

$\ddagger \quad$ Permanent address

[1] E. Feinberg, Nuovo Cimento A 34, 39 (1976); E. Shuryak, Phys. Lett. 79B, 135 (1978); E. Shuryak, Phys. Rep. 67 , 71 (1980); G. Domokos and J. I. Goldman, Phys. Rev. D 23, 203 (1981); G. Domokos, ibid 28, 123 (1983); K. Kajantie and H. I. Miettenen, Z. Phys. C 9, 341 (1981); 14 357, (1982); L. McLerran and T. Toimela, Phys. Rev. D 31, 545 (1985).

[2] For a recent review, see P.V. Ruuskanen in Particle Production in Highly Excited Matter, H.H. Gutbrod ed., Plenum, New York (1993), and references therein.

[3] J. Kapusta, P. Lichard and D. Seibert, Phys. Rev. D 44, 2774 (1991).

[4] C. Gale and J.I. Kapusta, Nucl. Phys. B357, 65 (1991).

[5] U.-G. Meißner, Phys. Rep. 161, 213 (1988), and references therein.

[6] Review of Particle Properties, Phys. Rev. D 45, (1992).

[7] P. Lichard, University of Minnesota preprint TPI-MINN-92/51-T, October 1992 (unpublished).

[8] P. Lichard and L. Van Hove, Phys. Lett. 245B, 605 (1990).

[9] P. Lichard and J.A. Thompson, Phys. Rev. D 44, 668 (1991).

[10] D. Bisello et al., Phys. Lett. 220B, 321 (1989), and references therein.

[11] N. Albrecht et al., Phys. Lett. 185B, 223 (1987), and references therein.

[12] M.E. Biagini, S. Dubnička, E. Etim and P. Kolář, Nuovo Cimento A 104, 363 (1991). The paper does not contain all the necessary parameters to reconstruct the form factors. We are indebted to Dr. Dubnička for providing us with a more detailed information in the form of ready-to-use computer codes.

[13] V.M. Budnev and V. A. Karnakov, Pisma Zh. Eks. Teor. Fiz. 29, 439 (1979).

[14] K. Kajantie, J. Kapusta, L. McLerran and A. Mekjian, Phys. Rev. D 34, 2476 (1986).

[15] K.K. Gudima, A.I. Titov and V.D. Toneev, Phys. Lett. 287B, 302 (1992); P. Koch, Z. Phys. C 57, 283 (1993).

[16] A. Shor, Phys. Lett. 233B, 231 (1989).

[17] J. Cleymans, K. Redlich, and H. Satz, Z. Phys. C 52, 517 (1991); K. Haglin, C. Gale, and V. Emel'yanov, Phys. Rev. D 46, 4082 (1992); 47, 973 (1993).

[18] L. Landau and I. Pomeranchuk, Dokl. Akad. Nauk SSSR 92, 535 (1953); 92, 735 (1953).

[19] J. Cleymans, V.V. Goloviznin, and K. Redlich, Phys. Rev. D 47, 989 (1993).

[20] L. Xiong, E.V. Shuryak and G.E. Brown, Phys. Rev. D 46, 3798 (1992).

[21] C. Song, University of Minnesota preprint NUC-MINN-93/2-T, January 1993 (unpublished). 


\section{FIGURES}

FIG. 1. The Feynman diagram for the radiative decay $\omega \rightarrow \pi^{0} \gamma$ in the model described in the text.

FIG. 2. A "generic" two-body amplitude with lepton pairs in the final state. The different $\{a, b\}$ combinations we consider are enumerated in the text. The vector meson $\mathrm{V}$ is chosen through isospin and $G$ parity arguments.

FIG. 3. Differential rate for lepton pair production via vector or pseudoscalar meson decay. The dashed line represents the contribution from $\omega \rightarrow \pi^{0} e^{+} e^{-}$, the dashed-dotted line is the rate from $\rho \rightarrow \pi e^{+} e^{-}$. The dotted line is the process $\phi \rightarrow \pi^{0} e^{+} e^{-}$. The structure in the latter channel is due to the $\rho(770)$. The solid line is the sum of all the decay processes, including those not listed in this caption but enumerated in the main text.

FIG. 4. Rate from PS + PS type reactions. The dashed line is the rate for the pion annihilation process. The dotted curve represents the contribution from $K^{+}+K^{-}$. The dashed-dotted line is the rate from $K^{0} \bar{K}^{0}$ annihilation. The solid line is the sum of the PS + PS processes.

FIG. 5. Rate from $\mathrm{V}+\mathrm{V}$ type reactions. The dashed curve is the $\rho^{+}+\rho^{-}$contribution. The dashed-dotted and dotted curves represent charged and neutral $K^{*}$ annihilation, respectively. The solid curve is the sum of the $\mathrm{V}+\mathrm{V}$ contributions.

FIG. 6. Rate from $\mathrm{V}+\mathrm{PS}$ type reactions. The dashed curve is the rate from $\omega+\pi^{0}$. The dashed-dotted curve is the contribution from $\rho+\pi$ and the dotted curve is the rate from $\rho^{0}+\eta$. Again, the solid line is a sum of all V + PS processes, as enumerated in the text.

FIG. 7. The solid line is the total rate at $T=150 \mathrm{MeV}$ from all processes discussed in the text. The dashed line is the pion-pion annihilation contribution only. The short-dashed curve represents the contribution from direct vector meson decays.

FIG. 8. Same caption as Fig. 7 but for the temperatures $T=100 \mathrm{MeV}$ (lower curves) and $T=200 \mathrm{MeV}$ (higher curves).

FIG. 9. The lepton pair momentum spectrum, $E d^{3} R / d^{3} p$ for lepton pair invariant masses between 1.1 and $3 \mathrm{GeV}$. This lower bound is chosen so as to exclude the $\phi$ peak. The full curve represents the contribution from all processes described in this work. The dashed curve is the pion-pion annihilation contribution only. 